\title{
Avoidable lethal torsade de pointes during pacemaker generator exchange caused by bepridil intoxication
}

\author{
Yoichi Ajiro ${ }^{1}$, Toshiharu Koike², Takashi Saito ${ }^{3}$, Fumiaki Mori ${ }^{3}$, and Kazunori Iwade ${ }^{3}$ \\ ${ }^{1}$ Niijuku Co-op Clinic \\ ${ }^{2}$ National Hospital Organisation Yokohama Medical Center \\ ${ }^{3}$ National Hospital Organization Yokohama Medical Center
}

September 14, 2020

\begin{abstract}
A 92-year-old small-framed man developed torsade de pointes during pacemaker-generator exchange. His family physician prescribed bepridil $200 \mathrm{mg}$ /day. High bepridil concentration of $1409 \mathrm{ng} / \mathrm{mL}$ was revealed. Electrophysiologists at pacemaker clinic should pay attention to arrhythmia management including bepridil concentration when the family physician prescribes bepridil to a pacemaker patient.
\end{abstract}

Avoidable lethal torsade de pointes during pacemaker generator exchange caused by bepridil intoxication

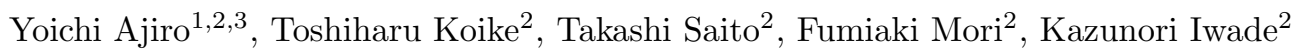

1. Division of Clinical Research, National Hospital Organization Yokohama Medical Center, Totsuka-ku, Yokohama, Japan

2. Department of Cardiology, National Hospital Organization Yokohama Medical Center, Totsuka-ku, Yokohama, Japan

3. Niijuku Co-op Clinic, Tokyo, Japan

Corresponding author: Yoichi Ajiro

Division of Clinical Research, National Hospital Organization Yokohama Medical Center; 3-60-2 Harajuku, Totsuka-ku, Yokohama, Kanagawa, 245-8575, Japan

Tel +81-45-851-2621; Fax +81-45-851-8316

E-mail:you617bacchus@gmail.com

Key Clinical Message

Regular or timely measurement of plasma bepridil concentration is important. Thus, an electrophysiologist at a pacemaker clinic should pay attention to arrhythmia management and pitfalls in hospital-clinic cooperation especially when the family physician prescribes bepridil to a pacemaker patient.

Abstract

A 92-year-old small-framed man developed torsade de pointes during pacemaker-generator exchange. His family physician prescribed bepridil $200 \mathrm{mg}$ /day. High bepridil concentration of $1409 \mathrm{ng} / \mathrm{mL}$ was revealed. Electrophysiologists at pacemaker clinic should pay attention to arrhythmia management including bepridil concentration when the family physician prescribes bepridil to a pacemaker patient. 
Keywords: torsade de pointes, pacemaker generator exchange, bepridil, intoxication, hospital-clinic cooperation

\section{Introduction}

Bepridil is used as an anti-arrhythmic agent because of its potassium-channel-blocking property, even though it was originally developed as an antianginal agent because of its calcium-channel-blocking property. ${ }^{1} \mathrm{Al}^{\mathrm{A}}$ though it has a potent inhibitory effect on atrial fibrillation, bepridil can cause lethal ventricular arrhythmias such as torsade de pointes (TdP). Therefore, atrial fibrillation management should be conducted carefully and adequately, measuring plasma bepridil concentration regularly or timely, when an atrial fibrillation patient is treated using bepridil. ${ }^{2}$ However, because atrial fibrillation is a common disease, the family physician often prescribes and manages arrhythmia. As regards pacemaker patients, because they visit the pacemaker clinic regularly, the responsibility of arrhythmia management might be obscured between the family physician and the electrophysiologist at the pacemaker clinic.

Herein, we report the case of a patient who had avoidable TdP during pacemaker generator exchange caused by bepridil intoxication.

\section{Patient Consent}

Written informed consent for publication was obtained from the patient and his guardian (son).

\section{Case History}

A 92-year-old man was admitted to our hospital for pacemaker generator exchange. He was small, with $150 \mathrm{~cm}$ in height and $43 \mathrm{~kg}$ in weight. A dual-chamber pacemaker (Identity, St. Jude Medical Japan, Tokyo, Japan) was implanted for sick sinus syndrome 12 years ago, and a daily dose of $200 \mathrm{mg}$ bepridil was started for associated paroxysmal atrial fibrillation by a general cardiologist in charge at that time. The bepridil prescription had been continued by his family physician. He visited the pacemaker clinic once every 6 months regularly and had not experienced any pacemaker-related problems. After 12 years of pacemaker usage, because the battery of the pacemaker had reached its elective replacement indicator, a scheduled pacemaker generator exchange was planned. The electrocardiogram at the pacemaker clinic showed atrial and ventricular pacing with corrected QT interval of $578 \mathrm{~ms}$, JT interval of $428 \mathrm{~ms}$, and QT dispersion of 109 ms (Fig. 1).

During the pacemaker generator exchange operation, QT prolongation occurred just after pacemaker reprograming to lower the heart rate setting (Fig. 2A). Subsequently, atrial fibrillation and TdP occurred (Fig. 2B). Fortunately, TdP was successfully terminated by $150 \mathrm{~J}$ defibrillation without any serious sequelae. The pacemaker generator exchange was completed using temporary backup pacing, which was immediately established after successful resuscitation. The serum potassium concentration was normal at $4.6 \mathrm{mEq} / \mathrm{L}$. Genetic screening showed no mutation in KCNQ1, KCNH2 , SCN5A,KCNE1, or HCN4 and a missense mutation in $L 897 T$, which was considered benign or likely benign. The plasma concentration of bepridil was high at $1409 \mathrm{ng} / \mathrm{mL}$. We concluded that TdP was caused by bepridil intoxication, and bepridil prescription was stopped. After cessation of bepridil prescription, the patient had been free from TdP for 45 months. Although the persistent atrial fibrillation became permanent atrial fibrillation, the patient had not experienced other adverse events since then.

\section{Discussion}

The present case highlighted two important issues: (1) the re-recognition of the seriousness of bepridil intoxication and importance of regular plasma bepridil concentration measurement; and (2) the importance of careful arrhythmia management by the family physician and electrophysiologist at the pacemaker clinic in pacemaker patients who are taking bepridil for atrial fibrillation.

Bepridil can cause lethal ventricular arrhythmias, such as TdP with an incidence rate of $0.4 \%-3.4 \%$, because of its multi-potassium-channel/calcium-channel-blocking property. ${ }^{3-8}$ Measurement of plasma bepridil concentration would be useful in preventing $\mathrm{TdP}$ as an adverse effect of bepridil use because a high plasma 
bepridil concentration of $>800 \mathrm{ng} / \mathrm{mL}$ is considered a risk factor for $\mathrm{TdP}^{2,}{ }^{5}$ Plasma bepridil concentration varies greatly among individuals, even at the same dosage, because of its complex pharmacokinetics. ${ }^{1,}{ }^{2,5}$ In addition, the treatment of bepridil intoxication can be difficult once adverse effects have occurred because bepridil has a very long elimination half-life of approximately $80 \mathrm{~h}$ after continued intake, which can be more prolonged due to less clearance in patients with low body weight and advanced age, like the present case. $^{1,5}$ In addition, cardiac pacing may mask electrocardiographic abnormalities especially by left bundle branch block, similar to configuration due to ventricular pacing. In the case of QT prolongation, the pacing may reduce QT prolongation itself and reduce the risk of TdP. ${ }^{9-15}$ Yasuda et al. reported that triggers for TdP among patients taking bepridil were older age, bradycardia, excessive baseline QT prolongation, and hypokalemia. ${ }^{3}$ In the present case, bradycardia by reprograming to lower the heart rate during pacemaker generator exchange operation and subsequent presentation of excessive QT prolongation due to bepridil intoxication would be direct triggers for TdP. Since bepridil has very long elimination half-life, the importance of regular plasma bepridil concentration measurement or timely measurement (for instance, prior to pacemaker generator exchange operation) in pacemaker patients is emphasized.

The present case may also indicate an important pitfall in the cooperation between the pacemaker clinic and family physician. Electrophysiologists at the pacemaker clinic may tend to neglect regular measurement of plasma bepridil concentration especially when the family physician prescribes bepridil. For the family physician, arrhythmia management might be a non-expert practice, so they might neglect regular measurement of plasma bepridil concentration, especially when the patient visits a cardiologist regularly like the present case. Considering that it is unlikely for the family physician to think of the fact that $200 \mathrm{mg}$ daily of bepridil is generally a risky dosage for small older patients, the electrophysiologist at the pacemaker clinic should pay attention to the arrhythmia management initiated by the family physician. The Swiss-cheese model, which is proposed as an organization's defense against accidents by stacking a series of barriers, is often adopted when considering patient safety management. From this perspective, following aspects would help evade this type of avoidable accident: (1) awareness of this type of pitfall; (2) awareness of the importance of regular measurement of plasma bepridil concentration especially at the pacemaker clinic; (3) awareness of the danger of bepridil intoxication and importance of regular measurement of plasma bepridil concentration by family physician, if possible, and (4) awareness of the importance of close cooperation between the electrophysiologist at the pacemaker clinic and family physician in charge of the arrhythmia management of the same patient. We hope that this case alerts both electrophysiologists at the pacemaker clinic and family physicians to the avoidable occurrence of fatal arrhythmia to enhance patient safety.

\section{Conclusion}

Regular or timely measurement of plasma bepridil concentration and careful close cooperation by the electrophysiologist and family physician in charge of arrhythmia management will contribute to patient safety by avoiding TdP in pacemaker patients taking bepridil.

Conflict of interest: No authors have conflicts of interest to declare.

Funding : This study was funded by the Clinical Research Division of National Hospital Organization Yokohama Medical Center.

Acknowledgements : We thank Editage (www.editage.jp) for English language editing.

\section{Author Contributions}

YA: Prepared the manuscript, acquired and interpreted data, and reviewed the literature.

TK: Acquisition of data and revised the manuscript.

TS, FM, KI: Revised the manuscript

\section{References}

1. Benet, L. Z. 1985. Pharmacokinetics and metabolism of bepridil. Am J Cardiol 55(7):8c-13c. 
2. Aonuma. K., Shiga, T., and H. Atarashi, et al. 2017. Guidelines for therapeutic drug monitoring of cardiovascular drugs clinical use of blood drug concentration monitoring (JCS 2015) - digest version. Circ J 81(4):581-612.

3. Yasuda, M., Nakazato, Y., and A. Sasaki, et al. 2006. Clinical evaluation of adverse effects during bepridil administration for atrial fibrillation and flutter. Circ J 70(6):662-666.

4. Yamashita, T., Ogawa, S., and T. Sato, et al. 2009. Dose-response effects of bepridil in patients with persistent atrial fibrillation monitored with transtelephonic electrocardiograms: a multicenter, randomized, placebo-controlled, double-blind study (J-BAF Study). Circ J 73(6):1020-1027.

5. Shiga, T., Suzuki, A., Naganuma, M., Hosaka, F., Shoda, M., and N. Hagiwara. 2011. Clinical outcome in patients with paroxysmal or persistent atrial fibrillation receiving bepridil. Circ J 75(6):1334-1342.

6. Harder, D. R., and N. Sperelakis. 1981. Bepridil blockade of Ca2+-dependent action potentials in vascular smooth muscle of dog coronary artery. J Cardiovasc Pharmacol 3(4):906-914.

7. Anno, T., Furuta, T., Itoh, M., Kodama, I., Toyama, J., and K. Yamada. 1984. Electromechanical effects of bepridil on rabbit isolated hearts. Br J Pharmacol 81(1):41-47.

8. Wang, J. C., Kiyosue, T., Kiriyama, K., and M. Arita. 1999. Bepridil differentially inhibits two delayed rectifier $\mathrm{K}(+)$ currents, $\mathrm{I}(\mathrm{Kr})$ and $\mathrm{I}(\mathrm{Ks})$, in guinea-pig ventricular myocytes. Br J Pharmacol 128(8):17331738.

9. Epstein, A. E., DiMarco, J. P., and K. A. Ellenbogen, et al. 2013. 2012 ACCF/AHA/HRS focused update incorporated into the ACCF/AHA/HRS 2008 guidelines for device-based therapy of cardiac rhythm abnormalities: a report of the American College of Cardiology Foundation/American Heart Association Task Force on Practice Guidelines and the Heart Rhythm Society. Circulation 127(3):e283-e352.

10. Eldar, M., Griffin, J. C., and J. A. Abbott, et al. 1987. Permanent cardiac pacing in patients with the long QT syndrome. J Am Coll Cardiol 10(3):600-607.

11. Eldar, M., Griffin, J. C., and G. F. Van Hare, et al. 1992. Combined use of beta-adrenergic blocking agents and long-term cardiac pacing for patients with the long QT syndrome. J Am Coll Cardiol 20(4):830837.

12. Oosterhoff, P., Thomsen, M. B., and J. N. Maas, et al. 2010. High-rate pacing reduces variability of repolarization and prevents repolarization-dependent arrhythmias in dogs with chronic AV block. J Cardiovasc Electrophysiol 21(12):1384-1391.

13. Keren, A., Tzivoni, D., Golhman, J. M., Corcos, P., Benhorin, J., and S. Stern. 1981. Ventricular pacing in atypical ventricular tachycardia. J Electrocardiol 14(2):201-205.

14. Tötterman, K. J., Turto, H., and T. Pellinen. 1982. Overdrive pacing as treatment of sotalol-induced ventricular tachyarrhythmias (torsade de pointes). Acta Med Scand Suppl 668:28-33.

15. Davison, E. T.. 1985. Amitriptyline-induced torsade de dointes. Successful therapy with atrial pacing. J Electrocardiol 18(3):299-301.

Figure Legends

Figure 1. 12-lead electrocardiogram at pacemaker clinic

Figure 2. Electrocardiogram and arterial blood pressure monitoring at the electrophysiology laboratory. A. QT prolongation just after pacemaker reprograming to lower heart rate setting. B. TdP occurrence and termination by $150 \mathrm{~J}$ defibrillation (black arrow). ABP: arterial blood pressure; TdP: torsade de pointes. 


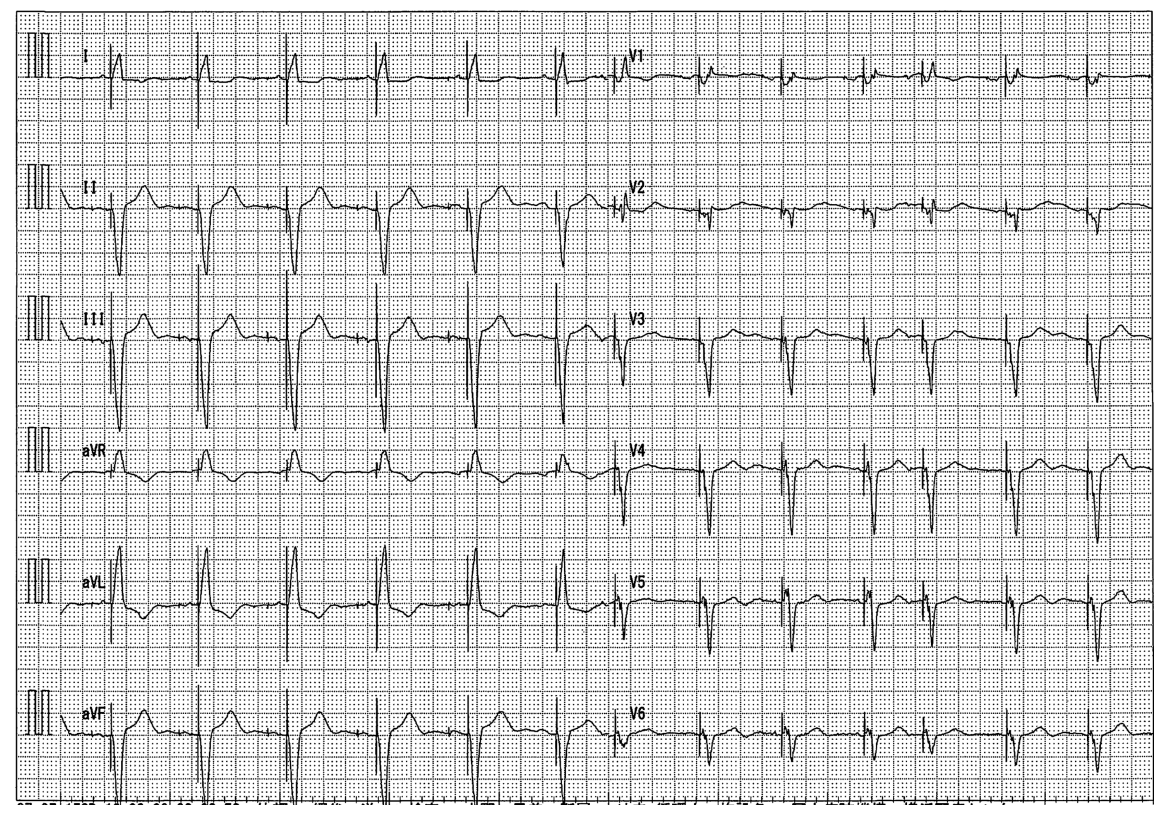

A

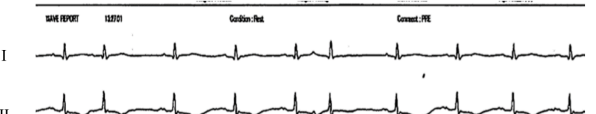

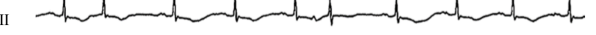

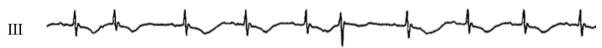

$\mathrm{aVR} \sim \gamma r$

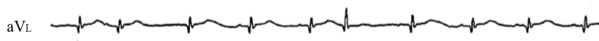



ABP

B 